\title{
Determinação Quantitativa da Concentração de Silicone em Antiespumantes por Espectroscopia FT-IR / ATR e Calibração Multivariada
}

\author{
Marcelo H. F. Garcia, Simone B. Farias, Bianca G. Ferreira \\ Poland Química Ltda., RJ
}

Resumo: Neste trabalho apresentamos uma alternativa para a dosagem do teor de silicone (polidimetilsiloxano) em antiespumantes por meio da técnica de espectroscopia no infravermelho com transformada de Fourier (FT-IR), com a utilização do acessório de reflectância total atenuada (ATR). Os espectros foram registrados na faixa espectral de 2500 a $780 \mathrm{~cm}^{-1}$, com resolução de $4 \mathrm{~cm}^{-1} \mathrm{e} 128$ varreduras. A calibração de um modelo linear por meio da utilização do método de mínimos quadrados parciais (PLS) aplicado aos espectros foi capaz de determinar satisfatoriamente a concentração de silicone nas amostras. Este método é de extrema importância para indústrias produtoras de antiespumantes siliconados, uma vez que o desempenho de tais produtos geralmente é avaliado em função da viscosidade dos mesmos. Muitas vezes no processo de fabricação de tais produtos ocorre uma homogeneização incompleta do silicone no solvente, o que leva a resultados de viscosidade que não são representativos das amostras analisadas. A determinação da concentração do teor de silicone é uma importante ferramenta para o Controle Estatístico de Processo (CEP), pois evita o desperdício de matérias-primas empregadas na fabricação dos antiespumantes.

Palavras-chave: Antiespumante, FT-IR, silicone, calibração multivariada.

\section{Quantitative Determination of Silicone in Antifoaming Products by FT-IR / ATR Spectroscopy and Multivariate Calibration}

Abstract: This work presents an alternative method to determine the concentration of silicone (polydimethylsiloxane) in antifoaming products using Fourier Transformed Infrared Spectroscopy (FT-IR) with the attenuated total reflectance (ATR) accessory. The spectra were recorded in the range from 2500 to $780 \mathrm{~cm}^{-1}$, with a resolution of $4 \mathrm{~cm}^{-1}$ and 128 scans. With calibration of a linear model using PLS regression method applied to spectral data we were able to determine the silicone concentration in the samples. This method may be useful for antifoaming producers since the performance of such products generally is evaluated as a function of their viscosity. Moreover, during manufacturing an incomplete homogenization of silicone in the solvent may occur, in which case the viscosity results are not representative of the samples. The determination of the silicone weight percentage is important to avoid losses in the production process and to help the Statistical Process Control.

Keywords: Antifoaming, FT-IR, silicone, multivariate calibration.

\section{Introdução}

O silicone é um material amplamente empregado na indústria química, sendo encontrado nos mais variados tipos de produtos e formulações. Pela analogia com as cetonas, em 1901 o nome silicone foi dado por Kipping aos compostos de fórmula genérica $\mathrm{R}_{2} \mathrm{SiO}$. Estes compostos foram rapidamente identificados como sendo poliméricos e atualmente correspondem aos polidialquilsiloxanos, de acordo com a fórmula representada na Figura 1.

$\mathrm{O}$ nome silicone foi adotado pela indústria e na maioria dos casos se refere a polímeros onde R é o radical metila (polidimetilsiloxano). Os radicais metila da cadeia podem ser substituídos por muitos outros grupos, tais como fenila, vinila ou trifluoropropila.

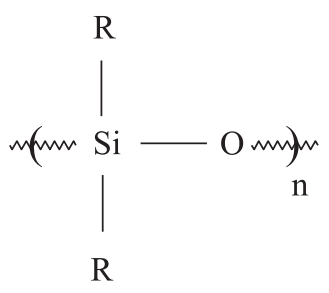

Figura 1. Estrutura do polialquildisiloxano

As propriedades do silicone que o tornam um material de grande importância para os mais diversos setores industriais são: excelente estabilidade térmica; boa resistência à radiação ultravioleta; atividade superficial; boas propriedades umectantes, anti-fricção e lubricidade; inércia hidrofóbica e fisiológica; estabilidade ao cisalhamento; excelentes proprie-

Autor para correspondência: Marcelo H. F. Garcia, Poland Química Ltda., Centro de Tecnologia Aplicada, Laboratórios de Controle de Qualidade e Analítica, Rua Capitão Guynemer 1080 - Xerém, CEP: 25241-390, Duque de Caxias, RJ. E-mail: marcelo.hilario@poland.com.br 
dades dielétricas; baixa volatilidade em altos pesos moleculares e alta volatilidade em baixos pesos moleculares ${ }^{[2]}$.

Devido à estrutura e natureza química do silicone, o controle de qualidade aplicado em sua fabricação encontra-se principalmente focado na determinação da viscosidade cinemática dos produtos finais, que está diretamente associada à sua massa molar ${ }^{[3,4]}$.

Antiespumantes a base de silicone vêm sendo empregados com certa freqüência em uma vasta gama de produtos, tais como fluidos de perfuração de poços de petróleo, lubrificantes, tintas e vernizes, etc. De uma forma geral, os antiespumantes são compostos obtidos a partir da diluição do silicone em um solvente apropriado para o uso final do produto ${ }^{[3,4]}$.

A técnica de espectroscopia no infravermelho tem sido amplamente utilizada na caracterização de substâncias e produtos químicos, e neste caso apresenta-se como uma alternativa interessante desde que os produtos analisados não apresentam água em sua composição, pois esta última tem forte absorção no infravermelho ${ }^{[6]}$. O advento da espectroscopia no infravermelho com Transformada de Fourier e dos microcomputadores, associados a novas técnicas, tais como as células de reflectância total atenuada, proporcionaram um considerável desenvolvimento na espectroscopia no infravermelho médio, tornando a mesma uma excelente alternativa em relação aos métodos tradicionais ${ }^{[5]}$. Os espectros obtidos, apesar de complexos, após passarem por um tratamento matemático adequado à aplicação do modelo de calibração, apresentam excelentes resultados.

Baseado nos fatos acima citados, este trabalho teve como objetivo construir um modelo de calibração para a determinação do teor de silicone em solvente orgânico (mistura de hidrocarbonetos predominantemente parafínicos) a fim de ser utilizado no controle de qualidade de antiespumantes à base de silicone.

\section{Materiais e Métodos}

\section{Amostras}

Para o modelo de calibração foram utilizadas vinte soluções padronizadas de silicone comercial proveniente da Dow Corning (Silicone Fluid 12500) e de solvente orgânico proveniente da Petrobrás S.A. (Solbrax ECO 255/285). As principais propriedades destes materiais são listadas a seguir.

Tabela 1. Propriedades físico-químicas do silicone e do solvente orgânico

\begin{tabular}{lc}
\hline \multicolumn{1}{c}{ Silicone Fluid 12500 } & Solbrax Eco 255/285 \\
\hline Polidimetilsiloxano & Querosene Desodorizado \\
Líquido Límpido Incolor & Cor Saybolt: 30 \\
Viscosidade Cinemática: 12500 & Ponto de Fulgor: $115^{\circ} \mathrm{C}$ \\
cSt & Densidade: $0,83 \mathrm{~g} / \mathrm{cm}^{3}$ \\
Densidade: $0,97 \mathrm{~g} / \mathrm{cm}^{3}$ & Ponto de Ebulição: \\
Ponto de Ebulição: $>65^{\circ} \mathrm{C}$ & $230^{\circ} \mathrm{C}$ (Inicial) $/ 280^{\circ} \mathrm{C}$ (Final) \\
\hline
\end{tabular}

Tabela 2. Concentrações das soluções-padrão de silicone utilizadas na calibração do modelo de regressão.

\begin{tabular}{cccc}
\hline Espectro & $\begin{array}{c}\text { Concentração } \\
(\mathbf{g} / \mathbf{1 0 0 g})\end{array}$ & Espectro & $\begin{array}{c}\text { Concentração } \\
(\mathbf{g} / \mathbf{1 0 0 g})\end{array}$ \\
\hline 1 & 20,0 & 14 & 33,0 \\
2 & 21,0 & 15 & 34,0 \\
3 & 22,0 & 16 & 35,0 \\
4 & 23,0 & 17 & 36,0 \\
5 & 24,0 & 18 & 37,0 \\
6 & 25,0 & 19 & 38,0 \\
7 & 26,0 & 20 & 39,0 \\
8 & 27,0 & 21 & 23,49 \\
9 & 28,0 & 22 & 25,79 \\
10 & 29,0 & 23 & 28,45 \\
11 & 30,0 & 24 & 32,63 \\
12 & 31,0 & 25 & 35,52 \\
13 & 32,0 & 26 & 38,65 \\
\hline & & & \\
\hline & 25,0 & 25 & \\
\hline
\end{tabular}

Após a calibração o modelo foi testado com seis soluções preparadas com as mesmas matérias-primas acima citadas, para determinar a validade do mesmo (Tabela 1 - Amostras de 1 a 20: Calibração; Amostras de 21 a 26: Validação).

\section{Análises por FT-IR}

Os espectros no FT-IR foram obtidos em um espectrômetro ThermoNicolet, modelo Avatar 330, equipado com cristal de reflectância de selenito de zinco. O cristal foi limpo entre as leituras das amostras com papel absorvente. Os espectros foram registrados na faixa espectral de 2500 a $780 \mathrm{~cm}^{-1}$, com resolução de $4 \mathrm{~cm}^{-1}$ para $\log (1 / \mathrm{R})$, onde $\mathrm{R}$ é a razão entre a intensidade de reflectância do background e da amostra. Para facilitar o entendimento dos dados, os sinais inicialmente obtidos como $\log (1 / \mathrm{R})$ foram registrados como valores de absorvâncias. Para cada espectro foram realizadas 128 varreduras, sendo que a combinação de todas as varreduras resulta em um espectro médio, representativo da amostra analisada. A escolha da faixa espectral acima citada foi baseada em dados da literatura sobre espectrometria de compostos orgânicos, sendo a faixa utilizada típica para análise de compostos contendo silício ${ }^{[6]}$.

\section{Análise e tratamento dos dados}

O modelo de regressão foi obtido por meio da utilização do método dos mínimos quadrados parciais (PLS - Partial Least Square), no qual as informações espectrais e as concentrações são utilizadas ao mesmo tempo para a calibração. Os espectros dos padrões são representados por uma matriz (X), que está relacionada a um vetor (y) que representa as concentrações das amostras pelas equações mostradas a seguir ${ }^{[7]}$ : 


$$
\begin{aligned}
& X=T P^{t}+E \\
& y=q t^{t}+f
\end{aligned}
$$

Os elementos das matrizes T são denominados de "scores" e os elementos de P e q os "loadings" de X e y respectivamente, e os elementos da matriz $\mathrm{E}$ e do vetor f representam os erros da modelagem de X e y. O índice t indica a operação de transposição de matrizes ${ }^{[7,8]}$.

Este procedimento tem como resultado uma equação linear de uma curva de calibração da seguinte forma:

$$
\mathrm{c}=\mathrm{Rb}+\mathrm{e}
$$

onde $\mathrm{R}$ é uma matriz de respostas para uma série de amostras, c é um vetor contendo a concentração de todas as amostras, b é o vetor que contém os parâmetros modelados e e é o vetor de erros. O vetor b é obtido da seguinte expressão:

$$
\mathrm{b}=\mathrm{W}\left(\mathrm{P}^{\mathrm{t}} \mathrm{W}\right)^{-1} \mathrm{q}^{\mathrm{t}}
$$

onde os vetores da matriz W são de tal forma que o produto da variância em X multiplicado pela correlação de XW com y é maximizado ${ }^{[7,8]}$.

Na utilização do método dos mínimos quadrados parciais (PLS) para a calibração do modelo, inicialmente foi realizada a determinação do número de componentes principais (variáveis latentes) para a construção do mesmo. Esta determinação foi realizada por meio do método de validação cruzada conhecido como "leave-one-out", onde a calibração é repetida $n$ vezes ( $n$ é o número de amostras utilizado para calibrar o modelo), e em cada repetição uma amostra do conjunto de calibração é tratada como uma amostra da previsão, ou seja, para cada uma das amostras o modelo calibrado é testado a fim de se determinar o número de componentes principais necessários para calibrar o modelo. $\mathrm{O}$ número adequado de componentes principais é aquele que apresenta a menor soma dos quadrados dos erros de previsão estimada (PRESS) ${ }^{[7-9]}$.

O desempenho do modelo foi representado pelo erro-padrão de previsão (SEP), obtido pela seguinte expressão:

$$
\mathrm{SEP}=\sqrt{\frac{\sum(C r-C p) 2}{n}}
$$

onde $\mathrm{Cr}=$ valores reais de concentração, $\mathrm{Cp}=$ valores previstos de concentração e $\mathrm{n}=$ número de amostras do conjunto de calibração. Por definição, a precisão da calibração é igual a duas vezes o erro padrão de previsão ${ }^{[10]}$.

\section{Resultados e Discussão}

Na Figura 2 são mostrados os 20 espectros obtidos na região de 2500 a $780 \mathrm{~cm}^{-1}$.

Todos os cálculos relativos aos parâmetros de calibração e validação do modelo foram realizados pelo software Matlab $^{[11]}$. A escolha deste software é justificada devido à sua interface amigável e pelo fato do mesmo apresentar um conjunto de funções pré-definidas que facilitam a construção de rotinas para a calibração multivariada pelo método PLS.

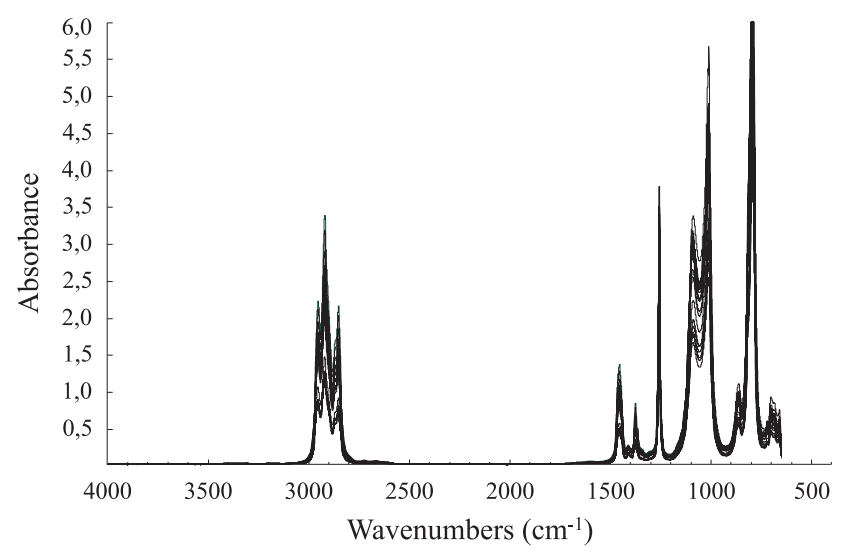

Figura 2. Espectros das soluções utilizadas na calibração do modelo de regressão linear.

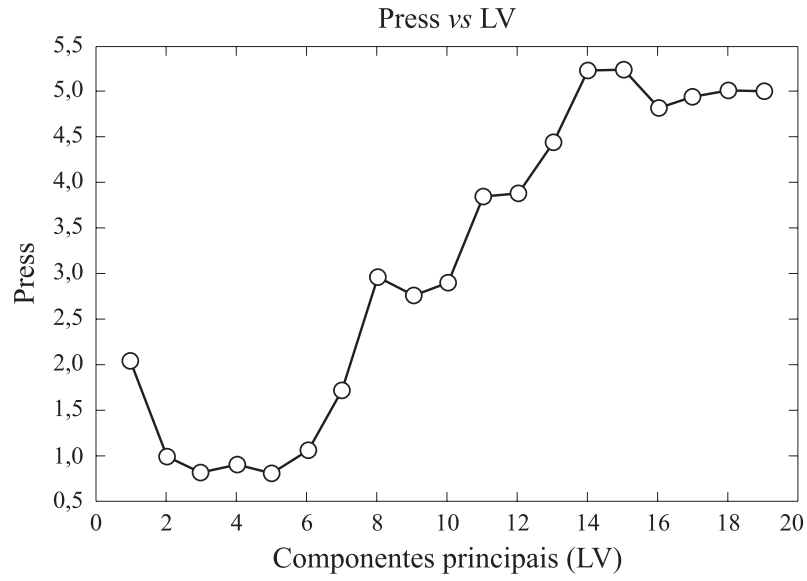

Figura 3. Gráfico de PRESS versus componentes principais (variáveis latentes - LV)

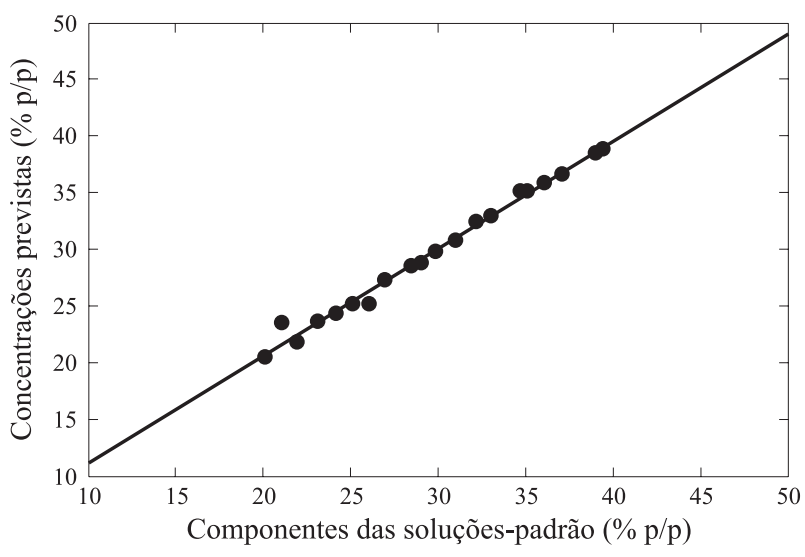

Figura 4. Reta de regressão do modelo de calibração

$\mathrm{O}$ número de componentes principais (variáveis latentes) necessário para estabelecer o modelo é mostrado na Figura 3. Verifica-se pelo gráfico que o número de componentes principais que apresenta o menor valor de PRESS é igual a três. A utilização de um número maior de componentes principais é desnecessária pois pelo próprio gráfico pode-se concluir que a capacidade de previsão do modelo diminui com o aumento do número das mesmas (devido ao aumento do valor de PRESS) ${ }^{[12]}$ 
Tabela 3. Validação do modelo obtido para determinação do teor de silicone

\begin{tabular}{cccc}
\hline Amostra & $\begin{array}{c}\text { Concentração } \\
\text { padrão } \\
(\% \mathbf{p} / \mathbf{p})\end{array}$ & $\begin{array}{c}\text { Concentração } \\
\text { prevista pelo modelo } \\
\text { de calibração } \\
(\mathbf{\%} \mathbf{p} / \mathbf{p})\end{array}$ & $\begin{array}{c}\text { Erro } \\
\text { percentual }\end{array}$ \\
\hline Teste1 & 23,49 & 23,42 & $-0,26$ \\
Teste2 & 25,79 & 25,48 & $-1,19$ \\
Teste3 & 28,45 & 28,06 & $-1,35$ \\
Teste4 & 32,63 & 32,91 & 0,85 \\
Teste5 & 35,52 & 35,78 & 0,73 \\
Teste6 & 38,65 & 38,37 & $-0,73$ \\
\hline
\end{tabular}

Na Figura 4 é apresentado o gráfico de comparação entre os valores utilizados para calibrar o modelo e os valores previstos para o mesmo.

$\mathrm{O}$ coeficiente de regressão $\left(\mathrm{R}^{2}\right)$ obtido para o modelo foi igual a 0.9952 , sendo o erro padrão de previsão (SEP) igual a $0,64 \% \mathrm{p} / \mathrm{p}$ e conseqüentemente a precisão da calibração igual a $1,29 \%$ p/p. Na Tabela 3 são mostrados os resultados previstos pelo modelo para as amostras teste e a comparação dos mesmos (através do cálculo do desvio percentual) com os valores padronizados das concentrações.

Verifica-se pelos valores obtidos para os erros percentuais que o modelo obtido prevê satisfatoriamente a concentração de silicone das amostras teste, uma vez que as mesmas apresentaram desvio percentual abaixo de $1,5 \%$.

\section{Conclusões}

O modelo de calibração obtido é capaz de prever com precisão de $1,290 \%$ os valores de concentração de silicone em antiespumantes, sendo o mesmo a partir de então utilizado como ferramenta no controle de qualidade da fabricação de tais produtos. Sua principal qualidade é o fato de ser capaz de controlar desperdícios de matéria-prima e assim auxiliar diretamente o controle estatístico de processos (CEP) realizado pelo laboratório de Controle de Qualidade.

Os resultados obtidos mostram a validade da técnica FT-IR/ATR e a utilização do acessório de reflectância total atenuada (ATR) associada à calibração multivariada (pelo método dos mínimos quadrados parciais) na previsão da con- centração de silicone em amostras de antiespumantes com razoável exatidão e precisão.

Assim, os antiespumantes fabricados a partir da calibração do modelo serão analisados pela técnica proposta com o objetivo de auxiliar o CEP realizado pelo Controle de Qualidade.

\section{Referências Bibliográficas}

1. Colas, A. - "Silicone Chemistry Overview", Dow-Corning Corporation, Michigan (1997)

2. Kuo, A. C. M. - "Polymer Data Handbook", Oxford University Press, Oxford (1999)

3. Harper, C. A. - “ Modern Plastics Handbook”, McGrawHill, New York (2000)

4. McGregor, R. R. - "Silicones and their uses", McGrawHill, London (1954)

5. Cadet, F. \& Offmann, B. - Spectrosc. Lett., 29 (3), pp. 523 - 526 (1996)

6. Silverstein, R. M.; Francis, X. - "Identificação Espectrofotométrica de Compostos Orgânicos", Editora LTC, Rio de Janeiro (2000)

7. Ferreira, M. M. C.; Antunes, A. M.; Melgo, M. S. \& Volpe, P.L. - Química Nova, 22, p. 724 (1999)

8. Ferreira, M. M. C.; Morgano, M. A,; Moryia, C. - "Brazilian Journal of Food Technology" - 6, pp. $77-83$ (2003)

9. Geladi, P. \& Kowalski, B. R. - Analitica Chimica Acta, 185, p.1 (1986)

10. Belon-Maurel, V.; Vallat, C. \& Goffinet, D. - Applied Spectroscopy, 49(5), p. 556 (1995)

11. MatLab - The Language of Technical Computing - version 6.5. The MathWorks Inc. (2001)

12. Sharaf, M. A.; Illman, D. L. \& Kowalski, B. R. "Chemometrics", John Willey \& Sons, New York (1986)

Recebido: 02/03/04

Reenviado: 28/06/04

Aprovado: 01/07/04 